\title{
Correction to: The ten amino acids of the oxygen-evolving enhancer of tobacco is sufficient as the peptide residues for protein transport to the chloroplast thylakoid
}

\author{
Sang Hoon Ma ${ }^{1} \cdot$ Hyun Min Kim ${ }^{1}$. Se Hee Park ${ }^{1}$. Seo Young Park ${ }^{1}$. Thanh Dat Mai ${ }^{1}$. Ju Hui Do ${ }^{1} \cdot$ Yeonjong Koo $^{2}$. \\ Young Hee Joung ${ }^{1}$ (1)
}

Published online: 1 February 2021

(c) The Author(s) 2021

\section{Correction to: Plant Molecular Biology \\ https://doi.org/10.1007/s11103-020-01106-8}

In the above mentioned publication, Fig. 5 was a duplicate of Fig. 4. The original article has been corrected and the proper version of Fig. 5 is also published here.

The original article can be found online at https://doi.org/10.1007/ s11103-020-01106-8.

Yeonjong Koo yeonjong@jnu.ac.kr

$\triangle$ Young Hee Joung yhjoung@jnu.ac.kr

1 School of Biological Science and Technology, Chonnam National University, Gwangju 61186, South Korea

2 Department of Agricultural Chemistry, Chonnam National University, Gwangju 61186, South Korea 
Fig. 5 The functional study of tTP1 peptide by serial deletion a Construction of recombinant plasmids carrying EGFPs fused with truncated tTP1. b Images are showing the fluorescence signals of EGFP directed by truncated tTP1s (GFP) and autofluorescence of chlorophyll to observe chlorophyll localization of TPs-EGFPs. Scale bars, $20 \mu \mathrm{m}$. c The protein size comparison of TPs-EGFPs extracted from the indicated transgenic plants. d Stroma and thylakoid of the chloroplast are extracted separately to determine the sub-organelle localization of TPs-EGFPs. Localization of Lhcb2 is indicated as a control of sub-organelle extractions. Lhcb2 visualization was performed for all panels repeatedly, and the same results as the representative were obtained.

The black arrowheads and white arrowheads indicate the GFP proteins that cleaved and uncleaved their signal peptides, respectively. CHL, chloroplast; STR, stroma; THY, thylakoid; THM, thylakoid membrane; THL, thylakoid lumen
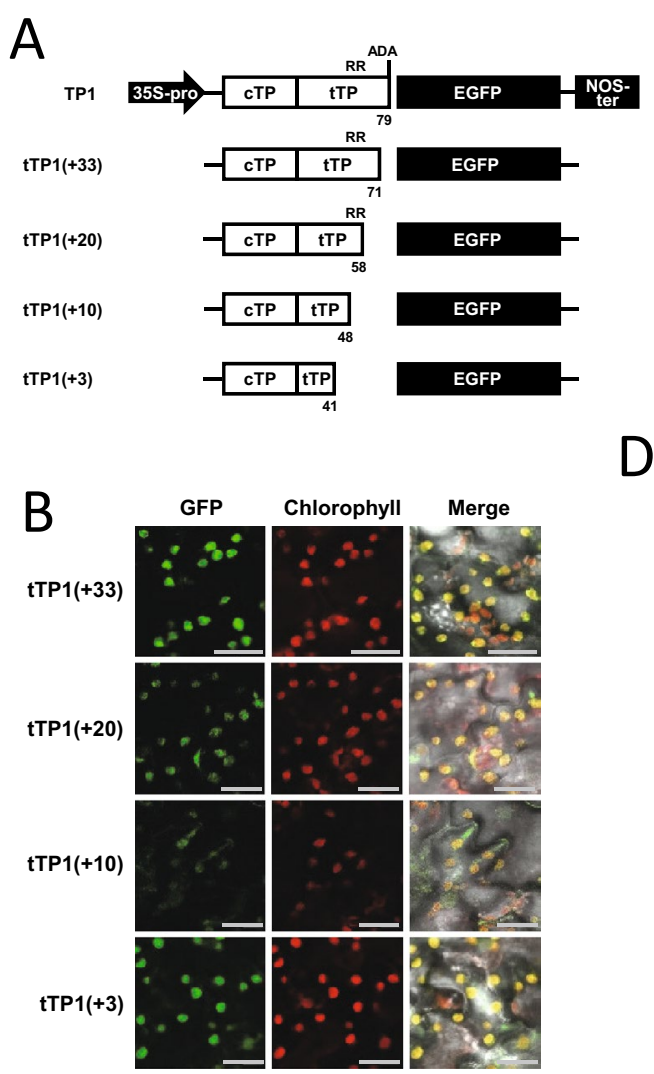

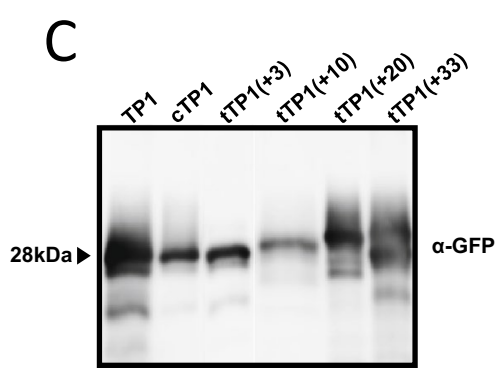

$D$

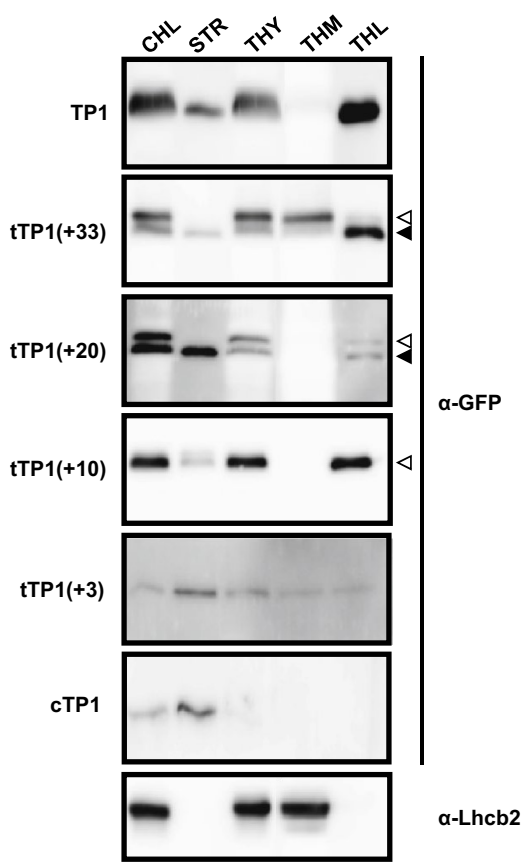

Publisher's Note Springer Nature remains neutral with regard to jurisdictional claims in published maps and institutional affiliations. 